\title{
REGION BASED 4D TOMOGRAPHIC IMAGE RECONSTRUCTION: APPLICATION TO CARDIAC X-RAY CT
}

\author{
G. Van Eyndhoven ${ }^{\star} \quad$ K.J. Batenburg ${ }^{\star \dagger} \quad$ J. Sijbers ${ }^{\star}$ \\ * iMinds-Vision Lab, University of Antwerp, B-2610 Antwerp (Wilrijk), Belgium \\ ${ }^{\dagger}$ Centrum Wiskunde \& Informatica, NL-1090 GB Amsterdam, The Netherlands
}

\begin{abstract}
$\mathrm{X}$-ray computed tomography (CT) is a powerful tool for noninvasive cardiac imaging. However, radiation dose is a major issue. In this paper, we propose an iterative reconstruction method that reduces the radiation dose without compromising image quality. This is achieved by exploiting prior knowledge in two ways: the reconstructed object is assumed to consist of both stationary and dynamic regions over time and the dynamic region is assumed to have sparse structures after a proper sparsifying space-time transform. Experiments on simulation data and a real $\mu \mathrm{CT}$ cardiac mouse dataset show that, with comparable image quality, the radiation dose can be substantially reduced compared to conventional acquisition/reconstruction protocols.
\end{abstract}

Index Terms - cardiac CT, iterative reconstruction, region based SIRT, dynamic CT, 4D CT, dose reduction

\section{INTRODUCTION}

Cardiac CT is a versatile tool for noninvasively visualizing the interior of the heart at different phases in the cardiac cycle. Its use ranges from preclinical studies [1] to the prediction of coronary artery disease and CT-assisted cardiac interventions [2].

To prevent motion artifacts in the reconstructed images, the heart is typically imaged at multiple phases of the cardiac cycle. A straightforward and well established approach is retrospective electrocardiogram (ECG) gating. In this technique, the heart is imaged multiple times at each angle while an ECG trace is recorded. Afterwards, the projection images are ordered according to their corresponding phase in the cardiac cycle and an image of the heart at each cardiac phase is reconstructed. Because multiple projection images are acquired at each scanning angle, radiation dose is a major issue. Previous studies report an estimated radiation dose of $12 \mathrm{mSv}$, which is the equivalent of 600 chest radiographs [3].

In a straightforward approach, radiation dose can be decreased simply by reducing the number of projections per phase bin. This will, however, give rise to limited data artifacts which may result in erroneous diagnosis or conclusions. A solution can be found in specific reconstruction algorithms, which allow for reducing the number of projections per phase bin without compromising image quality. Several methods have already been suggested in the literature.

A first class of methods regards the heart as a spatiotemporally $4 \mathrm{D}$ object in which the $3 \mathrm{D}$ reconstructions of the heart at different phases of the cardiac cycle are connected by a deformation model. If the deformation model is known a priori, it can be integrated into Filtered BackProjection (FBP) or Simultaneous Iterative Reconstruction Technique (SIRT) to produce artifact-free reconstructions $[4,5]$. Other techniques have been proposed to estimate the deformation parameters $[6,7]$, in case no a priori deformation model is available. These techniques, however, either assume rigid deformation or need high quality reconstructions of the different cardiac phases.

In a second class of methods, prior knowledge about the structure of the heart is assumed throughout the reconstruction process (e.g., sparseness in some transform domain). A standard approach consists of minimizing the total variation in the spatial and/or temporal domain $[8,9,10]$ or with respect to an a priori high quality reconstruction [11]. Other examples include the incorporation of a nonlocal means filter in the reconstruction algorithm [12].

In this contribution, we propose an iterative reconstruction algorithm that exploits two types of prior knowledge. Firstly, the reconstructed object is assumed to consist of stationary and dynamic regions. This is a realistic assumption for breath hold scanning protocols or in small animal imaging, where only the heart motion is relevant and the lung associated motion can often be neglected. This assumption leads to accurate reconstruction quality in the stationary region, without having to make any assumptions about its sparsity like the methods proposed in $[8,9,11,12]$. Secondly, the dynamic region is assumed to have sparse structures after a proper sparsifying space-time transformation. This leads to more accurate reconstruction quality in the dynamic region as well. Unlike the methods proposed in $[4,5,6,7]$, our method works without the need of estimating a deformation model, which is often error-prone and time-consuming. The proposed algorithm is validated with phantom data and applied to a real cardiac $\mu \mathrm{CT}$ dataset. 


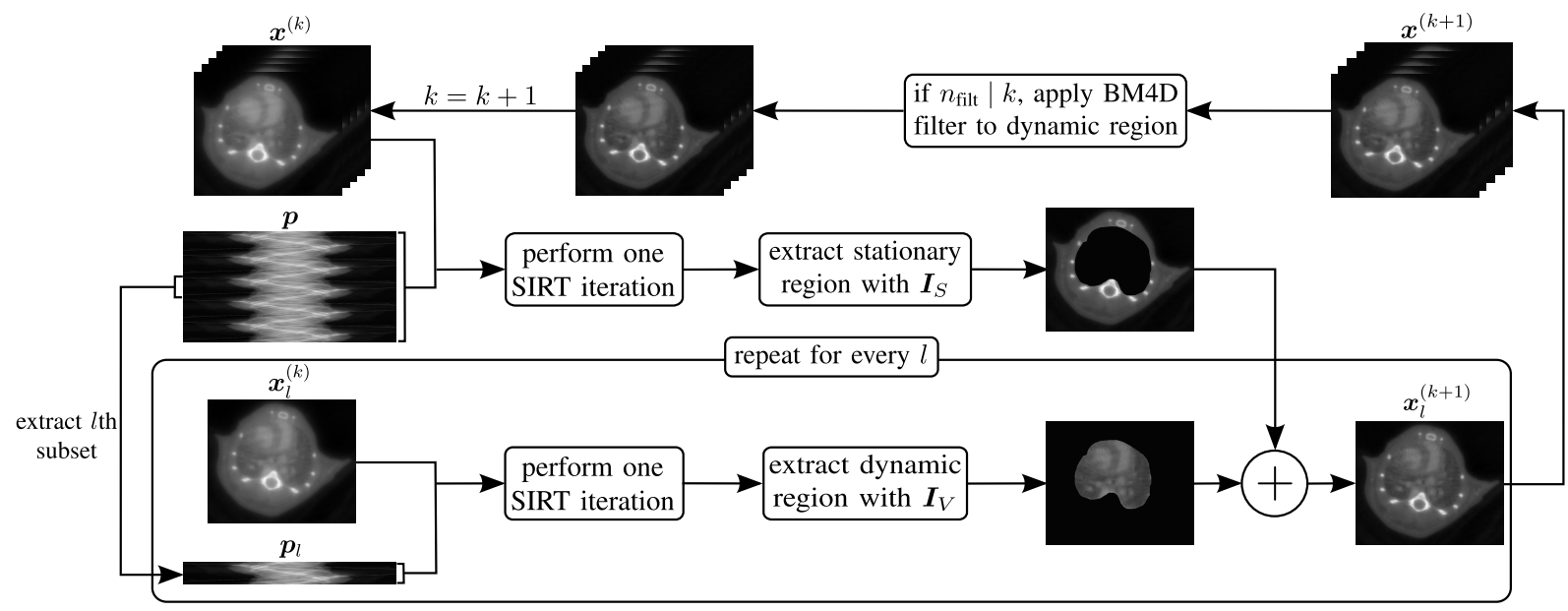

Fig. 1. Flowchart of the rSIRT-4D algorithm, illustrated with images from the cardiac mouse $\mu \mathrm{CT}$ dataset.

\section{METHOD}

In section 2.1, some notations and concepts of CT are introduced. Since this introduction is rather brief, the reader is referred to $[13,14]$ for more details. The proposed approach, named rSIRT-4D (region based dynamic SIRT), is explained in section 2.2.

\subsection{Notations and concepts}

Assume that the cardiac cycle is split up into $L$ phases by means of the ECG trace. For every $l=1, \ldots, L$, the reconstructed image of the scanned object at the $l^{\text {th }}$ phase bin is represented on a pixel grid consisting of $N$ pixels of which the pixel values are ordered in a column vector $\boldsymbol{x}_{l} \in \mathbb{R}^{N}$. The vector $\boldsymbol{p}_{l} \in \mathbb{R}^{M}$, referred to as the measured projection data, contains the log-corrected measured projection values ( $M$ in total) for the $l^{\text {th }}$ phase bin. Define $\boldsymbol{W}_{l} \in \mathbb{R}^{M \times N}$ as the forward projection matrix that models the data acquisition proces for the $l^{\text {th }}$ phase bin. The weight at the $j^{\text {th }}$ column and $i^{\text {th }}$ row of $\boldsymbol{W}_{l}$ represents the contribution of the $j^{\text {th }}$ pixel of $\boldsymbol{x}_{l}$ to the $i^{\text {th }}$ projection value of $\boldsymbol{p}_{l}$. Typically, the goal of algebraic reconstruction methods is to find $\boldsymbol{x}_{l}$ such that the projection distance $\left\|\boldsymbol{W}_{l} \boldsymbol{x}_{l}-\boldsymbol{p}_{l}\right\|$ is minimal for some norm $\|\cdot\|$. A well-established iterative method that minimizes the (weighted) projection distance is SIRT [15]. In SIRT, such a minimum is calculated starting from an initial reconstruction $\boldsymbol{x}_{l}^{(0)}=\mathbf{0}$ with the iterative process

$$
\boldsymbol{x}_{l}^{(k+1)}=\boldsymbol{x}_{l}^{(k)}+\boldsymbol{C}_{l} \boldsymbol{W}_{l}^{T} \boldsymbol{R}_{l}\left(\boldsymbol{p}_{l}-\boldsymbol{W}_{l} \boldsymbol{x}_{l}^{(k)}\right),
$$

where $\boldsymbol{R}_{l} \in \mathbb{R}^{M \times M}$ and $\boldsymbol{C}_{l} \in \mathbb{R}^{N \times N}$ are defined as the diagonal matrices with inverse row sums and inverse column sums of $\boldsymbol{W}_{l}$, respectively. Finally, define $\boldsymbol{p}=$ $\left(\boldsymbol{p}_{1}^{T}, \boldsymbol{p}_{2}^{T}, \ldots, \boldsymbol{p}_{L}^{T}\right)^{T} \in \mathbb{R}^{L M}$ as the vertical concatenation of all projection data, $\boldsymbol{x}=\left(\boldsymbol{x}_{1}^{T}, \boldsymbol{x}_{2}^{T}, \ldots, \boldsymbol{x}_{L}^{T}\right)^{T} \in \mathbb{R}^{L N}$ as the vertical concatenation of all phase bin reconstructions and $\boldsymbol{W}$ as the block diagonal matrix consisting of blocks
$\boldsymbol{W}_{1}, \boldsymbol{W}_{2}, \ldots, \boldsymbol{W}_{L}$. The projection distance for the full problem is defined as $\|\boldsymbol{W} \boldsymbol{x}-\boldsymbol{p}\|$. In low-dose protocols where the number of projection images per phase bin is low, directly minimizing $\|\boldsymbol{W} \boldsymbol{x}-\boldsymbol{p}\|$ for $\boldsymbol{x}$ (e.g., by applying SIRT separately on each projection data subset $\boldsymbol{p}_{l}$ ) will typically result in a solution $\boldsymbol{x}$ that is fully dominated by noise. However, by imposing constraints on the solution $\boldsymbol{x}$, the set of possible solutions becomes smaller and the final solution is typically less influenced by artifacts and noise. This approach is followed in rSIRT-4D, where the reconstruction is assumed to consist of stationary and dynamic regions over time and a sparsifying space-time transformation sets a restriction on the pixels in the dynamic region.

\section{2. rSIRT-4D}

The proposed rSIRT-4D algorithm is visualized with the flowchart in Fig. 1. Let $\boldsymbol{I}_{V} \in\{0,1\}^{N \times N}$ be the binary diagonal matrix representing the operator that sets all pixels belonging to the stationary region to 0 , i.e., $\left(\boldsymbol{I}_{V} \boldsymbol{x}\right)_{i}=0$ if $i$ is a pixel index corresponding to the stationary region and $\left(\boldsymbol{I}_{V} \boldsymbol{x}\right)_{i}=(\boldsymbol{x})_{i}$ otherwise. Analogously, the binary diagonal matrix $\boldsymbol{I}_{S} \in\{0,1\}^{N \times N}$ is defined as the operator setting all pixels in the dynamic region to 0 . Furthermore, define $\boldsymbol{R} \in \mathbb{R}^{L M \times L M}$ and $\boldsymbol{C} \in \mathbb{R}^{N \times N}$ as the diagonal matrices with inverse row sums and inverse column sums of $\boldsymbol{W}$, respectively. The rSIRT-4D algorithm can now be formulated as the iterative process in Algorithm 1. From the flowchart in Fig. 1 and code line 5 in Algorithm 1, one can notice that the inner most loop calculates two updates: A traditional SIRT update for the stationary region using all available projection data $\boldsymbol{p}$ and an update for the dynamic region using only the projection data corresponding to the relevant phase bin [16]. Since the update for the stationary region is based on all available projection data $\boldsymbol{p}$, image quality in the stationary region greatly improves. Also, pixels in the stationary region are forced to be the same at all phase bins, thereby reducing the 


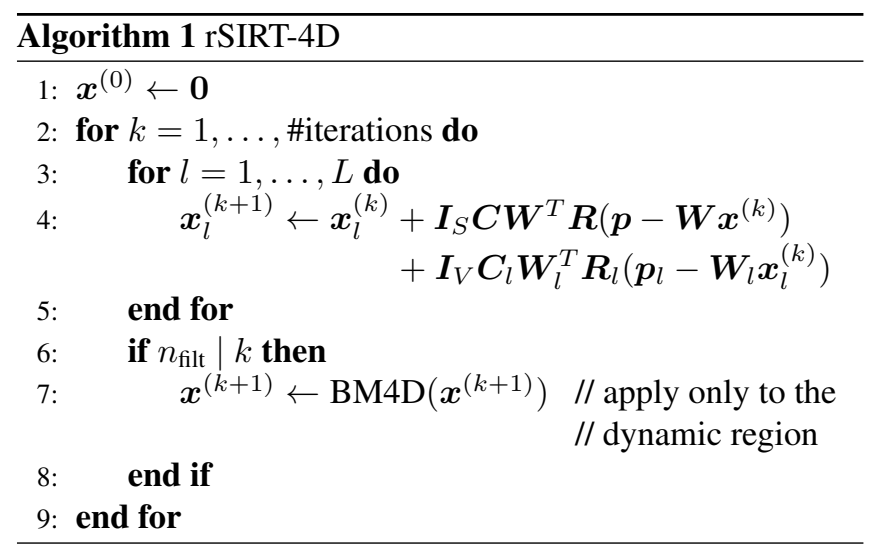

size of the solution space. Because the update for the dynamic region is computed using the projection difference of the previous estimate, this also improves the image quality in the dynamic region to some extent. To further enhance the quality in the dynamic region, a spatial-temporal block matching filter (BM4D), as described in [17], is applied to the dynamic region, at every $n_{\text {filt }}$ iterations. The forward and backward projection operations were implemented with the ASTRA toolbox [18].

\section{EXPERIMENTS}

In this section, the rSIRT-4D method is validated with both simulation (Section 3.1) and real data (Section 3.2) experiments. As a validation measure, the relative root mean squared error (RRMSE) is utilized. The rSIRT-4D reconstruction is compared to the standard SIRT and FBP reconstruction (calculated independently for each phase bin), to the rSIRT reconstruction [16] and to the SIRT reconstruction where at each $n_{\text {filt }}$ iterations the BM4D filter is applied to the entire reconstruction domain. The latter is referred to as SIRT-BM4D. For all algorithms and subsequent experiments, the parameter value $n_{\text {filt }}=20$ was chosen. The dynamic region, for which a mask is needed in rSIRT and rSIRT-4D, was indicated manually. For automatic detection methods the reader is referred to [16].

\subsection{Experiment with the XCAT phantom}

A first validation of the proposed method is performed with the XCAT human simulation phantom [19]. A breath holding protocol was assumed, hence only the motion induced by the heart is considered. An online synchronization scanning approach was simulated, i.e., projections corresponding to each phase bin were generated subsequently by simulating radiographs only if the ECG signal is at a specific phase. Furthermore, the projection angles associated with each phase bin projection dataset were shifted with a small sub-angular shift, to assure that no projection angle is sampled twice. The projections were simulated with a parallel beam geometry and
Poisson distributed noise was applied assuming an incoming beam intensity of $4 \times 10^{4}$ (photon count). Apart from FBP, each reconstruction method was applied with 200 iterations.

\subsection{Cardiac mouse dataset}

A cardiac mouse dataset was acquired with a SkyScan 1176 $\mu \mathrm{CT}$ scanner. It contains projection images of a mouse's thorax at 515 equiangular directions over a full $360^{\circ}$ range. At each angle, a total of 20 projection images were captured. An ECG signal was measured simultaneously. Projections were retrospectively ordered in 5 phase bins according to the periodic ECG signal. If multiple images were assigned to a bin at a certain projection angle, the images were averaged. If no image could be assigned to a bin at a certain projection angle, the projection image of the nearest angular neighbor was assigned to the bin.

Define $\left\{\theta_{i}\right\}_{i=1, \ldots, 515}$ as the ordered set of equiangular projection directions. For each phase bin, we selected $1 / 5^{\text {th }}$ of the available projection images, defined by the set of angles $\Omega_{j}=\left\{\theta_{i} \mid i \bmod 5=j\right\}$ where $j$ represents the phase bin index, i.e., $j=1, \ldots, 5$. This reduced dataset can be regarded as a dataset obtained with an online synchronization method. Note that this procedure would expose the scanned animal to only $20 \%$ of the radiation that was applied for acquiring the full dataset. All reconstruction methods are applied on this reduced projection data set and compared to a ground truth image (see Fig. 5(a)) that was generated by the application of SIRT on the full projection dataset.

\section{RESULTS}

\subsection{Experiment with the XCAT phantom}

The experiment with the XCAT phantom was repeated for a varying number of projections per phase bin. The RRMSE as a function of the number of projections per phase bin is displayed in Fig. 2. The reconstructions based on 30 projections per phase bin can be observed in Fig. 3. It is clear that, especially if few projections are available per phase bin, rSIRT-4D outperforms all other reconstruction methods with respect to the RRMSE metric.

\subsection{Cardiac mouse dataset}

The RRMSE as a function of iteration number is displayed in Fig. 4. A good trade-off between reconstruction quality in the dynamic (see Fig.4(c)) and in the stationary region (see Fig.4(b)) can be found at 200 iterations for all methods. The corresponding reconstructions of the last phase bin are displayed in Fig. 5. Both the dynamic and the stationary region are more accurately reconstructed with rSIRT-4D with respect to all other reconstruction methods, including SIRT-BM4D. Especially the fine structures are more accurately reconstructed by rSIRT-4D (see Fig. 5(e)) in comparison to SIRT-BM4D (see Fig. 5(c)). 


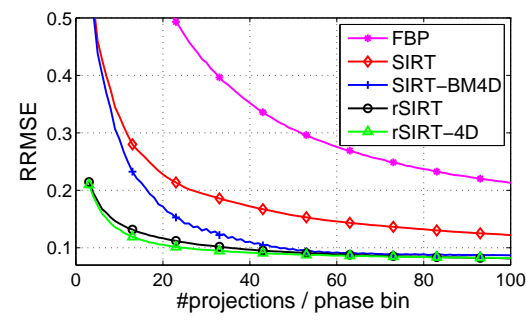

(a) Full reconstruction domain

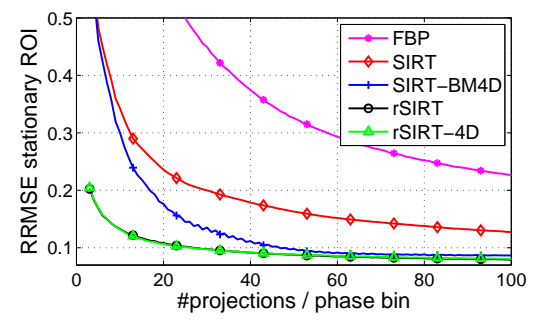

(b) Stationary ROI

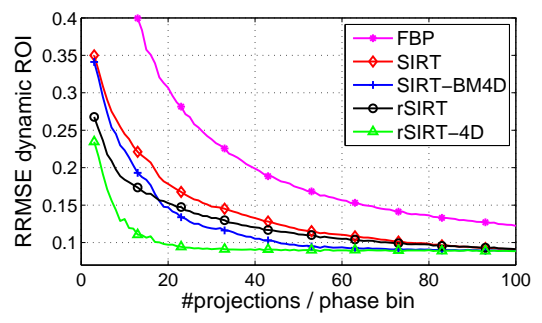

(c) Dynamic ROI

Fig. 2. The RRMSE in function of the number of projections per phase bin for the XCAT phantom.

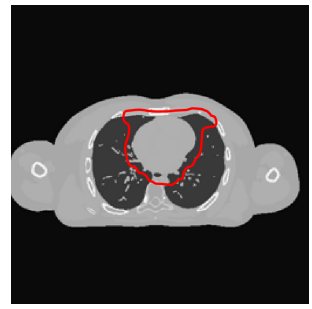

(a) Ground truth

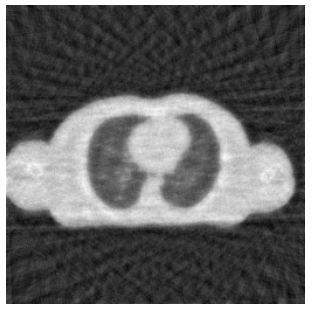

(b) SIRT

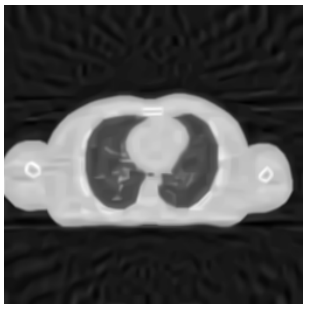

(c) SIRT-BM4D

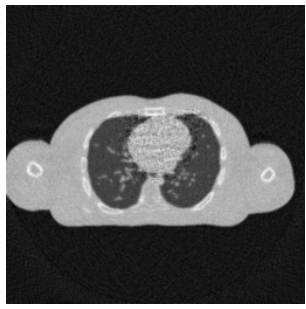

(d) rSIRT

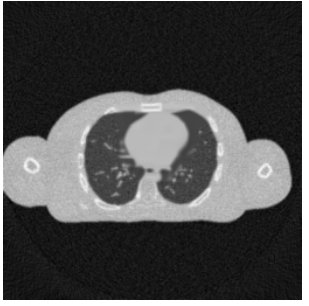

(e) rSIRT-4D

Fig. 3. Ground truth (the border between static and dynamic region is indicated with a red curve) and reconstructions of the $5^{\text {th }}$ phase bin of the XCAT phantom generated by the different algorithms with 200 iterations. For each phase bin, 30 projections were simulated.

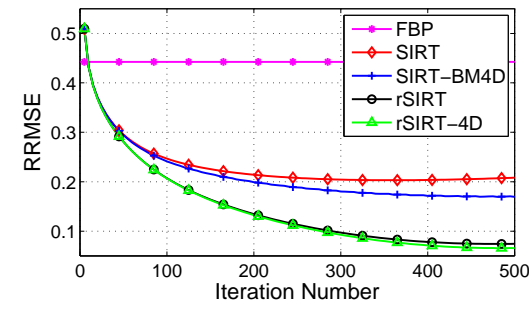

(a) Full reconstruction domain

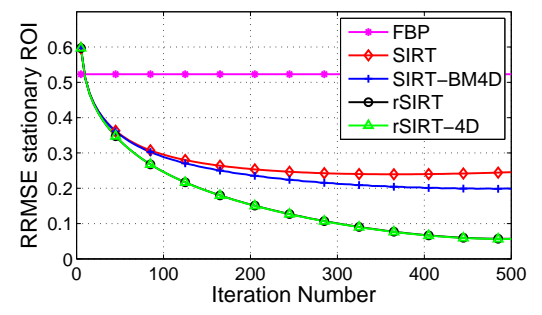

(b) Stationary ROI

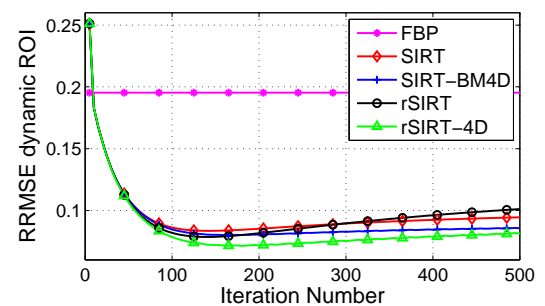

(c) Dynamic ROI

Fig. 4. The RRMSE in function of the iteration number for the cardiac mouse $\mu \mathrm{CT}$ dataset.

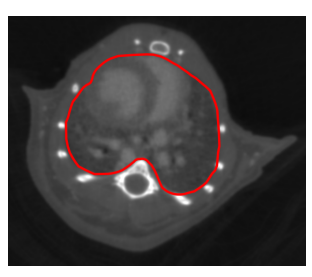

(a) Ground truth

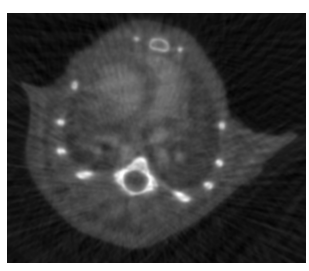

(b) SIRT

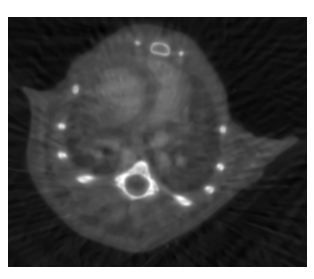

(c) SIRT-BM4D

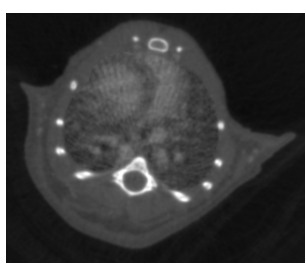

(d) rSIRT

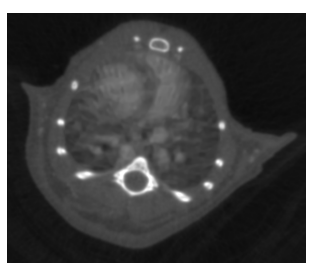

(e) rSIRT-4D

Fig. 5. Ground truth (the border between static and dynamic region is indicated with a red curve) and reconstructions of the $5^{\text {th }}$ phase bin of the cardiac mouse dataset generated by the different algorithms with 200 iterations.

\section{CONCLUSION}

The proposed rSIRT-4D method is able to reconstruct 4D images of comparable quality with respect to conventional methods, using fewer projection data. Using the same amount of data, rSIRT-4D provides substantially improved reconstruction quality in the stationary and dynamic region.

\section{ACKNOWLEDGEMENT}

The authors would like to thank Xuan Liu and Alexander Sasov (Bruker-MicroCT, Belgium) for the SkyScan 1176 $\mu \mathrm{CT}$ dataset. Networking support was provided by the EXTREMA COST Action MP1207. 


\section{REFERENCES}

[1] C. T. Badea, M. Drangova, D. W. Holdsworth, and G. A. Johnson, "In vivo small-animal imaging using microCT and digital subtraction angiography," Phys. Med. Biol., vol. 53, no. 19, pp. R319-R350, 2008.

[2] K. Nieman, "Cardiac CT in clinical practice," in Cardiac CT, pp. 31-39. Springer, 2014.

[3] J. Hausleiter, T. Meyer, F. Hermann, M. Hadamitzky, M. Krebs, T. C. Gerber, C. McCollough, S. Martinoff, A. Kastrati, A. Schömig, and S. Achenbach, "Estimated radiation dose associated with cardiac CT angiography," JAMA, vol. 301, no. 5, pp. 500-507, 2009.

[4] G. Van Eyndhoven, J. Sijbers, and K. J. Batenburg, "Combined motion estimation and reconstruction in tomography," in ECCV 2012 Ws/Demos, 2012, vol. 7583, pp. 12-21.

[5] T. Li, E. Schreibmann, Y. Yang, and L. Xing, "Motion correction for improved target localization with onboard cone-beam computed tomography," Phys. Med. Biol., vol. 51, no. 2, pp. 253-267, Jan. 2006.

[6] U. van Stevendaal, J. von Berg, C. Lorenz, and M. Grass, "A motion-compensated scheme for helical cone-beam reconstruction in cardiac CT angiography," Med. Phys., vol. 35, no. 7, pp. 3239-3251, 2008.

[7] M. Prummer, J. Hornegger, G. Lauritsch, L. Wigstrom, E. Girard-Hughes, and R. Fahrig, "Cardiac c-arm CT: a unified framework for motion estimation and dynamic CT," IEEE Trans. Med. Imag., vol. 28, no. 11, pp. 18361849, 2009.

[8] J. Song, Q. H. Liu, G. A. Johnson, and C. T. Badea, "Sparseness prior based iterative image reconstruction for retrospectively gated cardiac micro-CT," Med. Phys., vol. 34, no. 11, pp. 4476-4483, 2007.

[9] L. Ritschl, S. Sawall, M. Knaup, A. Hess, and M. Kachelrieß, "Iterative 4D cardiac $\mu \mathrm{CT}$ image reconstruction using an adaptive spatio-temporal sparsity prior," Phys. Med. Biol., vol. 57, no. 6, pp. 1517, 2012.

[10] C. Mory, V. Auvray, B. Zhang, M. Grass, D. Schäfer, S. J. Chen, J. D. Carroll, S. Rit, F. Peyrin, P. Douek, and L. Boussel, "Cardiac C-arm computed tomography using a 3D+time ROI reconstruction method with spatial and temporal regularization," Med. Phys., vol. 41, no. 2, pp. 021903, 2014.

[11] G. Chen, J. Tang, and S. Leng, "Prior image constrained compressed sensing (PICCS): A method to accurately reconstruct dynamic CT images from highly undersampled projection data sets," Med. Phys., vol. 35, no. 2, pp. 660-663, 2008.
[12] Z. Tian, X. Jia, B. Dong, Y. Lou, and S. B. Jiang, "Low-dose 4DCT reconstruction via temporal nonlocal means," Med. Phys., vol. 38, no. 3, pp. 1359-1365, 2011.

[13] T. M. Buzug, Computed Tomography: From Photon Statistics to Modern Cone-Beam CT, Springer-Verlag, 2008.

[14] A. C. Kak and S. Malcolm, Principles of computerized tomographic imaging, Society of Industrial and Applied Mathematics, 2001.

[15] J. Gregor and T. Benson, "Computational analysis and improvement of SIRT.," IEEE Trans. Med. Imag., vol. 27, no. 7, pp. 918-924, 2008.

[16] G. Van Eyndhoven, K. J. Batenburg, and J. Sijbers, "Region-based iterative reconstruction of structurally changing objects in CT," IEEE Trans. Imag. Proc., vol. 23, no. 2, pp. 909-919, 2014.

[17] M. Maggioni, V. Katkovnik, K. Egiazarian, and A. Foi, "Nonlocal transform-domain filter for volumetric data denoising and reconstruction," IEEE Trans. Imag. Proc., vol. 22, no. 1, pp. 119-133, Jan 2013.

[18] W. J. Palenstijn, K. J. Batenburg, and J. Sijbers, "Performance improvements for iterative electron tomography reconstruction using graphics processing units (GPUs)," J. Struct. Biol., vol. 176, no. 2, pp. 250-253, 2011.

[19] W. P. Segars, M. Mahesh, T. J. Beck, E. C. Frey, and B. M. Tsui, "Realistic CT simulation using the 4D XCAT phantom," Med. Phys., vol. 35, no. 8, pp. 38003808, 2008. 